INGENIERÍA DE ALIMENTOS

\title{
Parámetros cinéticos de transferencia de masa durante el freído por inmersión de rodajas de yuca (Manihot esculenta Crantz)
}

INGENIERÍA DE ALIMENTOS

\section{Kinetic parameters of mass transfer in deep-fat frying of cassava slices (Manihot esculenta Crantz)}

\author{
Fabián A. Ortega*§, Everaldo J. Montes* \\ *Programa de Maestría en Ciencias Agroalimentarias, Universidad de Córdoba, Montería, Colombia \\ §ingenierofabianortega@gmail.com,everaldomontes@yahoo.com
}

Recibido: Agosto 5 de 2013 - Aceptado: Mayo 23 de 2014

\begin{abstract}
Resumen
Los cambios de humedad y absorción de aceite ocurridos en el freído por inmersión de rodajas de yuca (Manihot esculenta Crantz) variedad ICA-costeña fueron evaluados con el fin de determinar los parámetros cinéticos de absorción de aceite y pérdida de humedad. Rodajas de $3.5 \mathrm{~cm}$ de diámetro y $1.3 \mathrm{~mm}$ de espesor fueron pretratadas así: (i) escaldado a $80^{\circ} \mathrm{C}$ por $4 \mathrm{~min}$; (ii) osmodeshidratación en solución salina $(3 \% \mathrm{p} / \mathrm{p})$ a $30^{\circ} \mathrm{C}$ por 4 horas y relación 1:20 en peso. Las rodajas fueron procesadas en aceite de palma Caribe ${ }^{\circledR}$ a $140^{\circ} \mathrm{C}, 160^{\circ} \mathrm{C}$ y $180^{\circ} \mathrm{C}$ en una relación de $0.005 \mathrm{~kg}$ muestra $/ \mathrm{L}$ de aceite. El experimento fue conducido bajo un diseño en bloques completamente aleatorizado con arreglo factorial $3^{2}$ (factores: temperatura y pre-tratamientos) con tres repeticiones. Los contenidos de humedad y aceite se determinaron aplicando los métodos AOAC 984.25/2002 y Soxhlet, respectivamente. La difusividad efectiva de humedad tuvo valores entre $10.44 \times 10^{-9}$ y $148 \times 10^{-9} \mathrm{~m}^{2} / \mathrm{s}$. El coeficiente convectivo de transferencia de humedad tuvo valores entre $1.37 \times 10^{-5}$ y $7.37 \times 10^{-5} \mathrm{~m} / \mathrm{s}$ con un comportamiento lineal con respecto a la temperatura del aceite. El contenido de aceite absorbido en el equilibrio estuvo entre 0.1391 y $0.3607 \mathrm{~g}$ aceite/g sólidos y las velocidades específicas entre $8.14 \times 10^{-3}$ y $77.08 \times 10^{-3} \mathrm{~s}^{-1}$. El escaldado y la osmodeshidratación afectaron significativamente a la difusividad efectiva de transferencia de masa y el contenido de aceite en el equilibrio $(\mathrm{p}<0.05)$.
\end{abstract}

Palabras clave: Aceite absorbido, Coeficiente convectivo, Difusividad efectiva, Ley de Fick.

\begin{abstract}
Moisture and oil absorption changes occurred in the deep-fat frying of cassava slices (Manihot esculenta Crantz), ICAcosteña variety, were evaluated to determine the kinetic parameters of oil absorption and moisture loss. Slices with $3.5 \mathrm{~cm}$ diameter and $1.3 \mathrm{~mm}$ thick were pretreated with (i) blanching at $80^{\circ} \mathrm{C}$ for $4 \mathrm{~min}$, (ii) osmotic dehydration in saline solution $(3 \% \mathrm{w} / \mathrm{w})$ at $30^{\circ} \mathrm{C}$ for 4 hours and a ratio of $1: 20$ by weight. The slices were processed in Caribbean ${ }^{\circledR}$ palm oil at $140^{\circ} \mathrm{C}, 160^{\circ} \mathrm{C}$ and $180^{\circ} \mathrm{C}$ and a ratio of $0.005 \mathrm{~kg}$ sample / $\mathrm{L}$ oil. The experiment was conducted under a randomized completely block design with factorial arrangement $3^{2}$ (factors: temperature and pretreatment) with three replications. The moisture and oil contents were determined by AOAC 984.25/2002 and Soxhlet methods, respectively. Moisture's effective diffusivity ranged between $10.44 \times 10^{-9}$ and $148 \times 10^{-9} \mathrm{~m}^{2} / \mathrm{s}$. The convective moisture transfer coefficient ranged between $1.37 \times 10^{-5}$ y $7.37 \times 10^{-5} \mathrm{~m} / \mathrm{s}$ having a linear behavior with respect to the oil temperature. In the equilibrium, the oil absorption content ranged between 0.1391 and $0.3607 \mathrm{~g}$ oil / $\mathrm{g}$ solids and the specific rates between $8.14 \times 10^{-3}$ y $77.08 \times 10^{-3} \mathrm{~s}^{-1}$. Blanching and osmotic dehydration significantly affected the effective diffusivity of mass transfer and the oil content at equilibrium $(\mathrm{p}<0.05)$.
\end{abstract}

Keywords: Oil absorbed, Convective coefficient, Effective diffusivity, Fick's law. 


\section{Introducción}

El freído por inmersión puede ser definido como un proceso de cocción y secado en el cual el producto está en contacto con un aceite caliente a una temperatura por encima del punto de ebullición del agua. Las temperaturas de freído oscilan entre $130^{\circ} \mathrm{C}$ y $190^{\circ} \mathrm{C}$, pero las temperaturas más comunes están entre $170^{\circ} \mathrm{C}$ y $190^{\circ} \mathrm{C}$ (Yamsaengsung \& Moreira, 2002; Bassama et al., 2012; Vitrac et al., 2000).

Como una de las consecuencias de la transferencia de calor desde el aceite hasta el alimento y la transferencia de masa entre el aceite y el alimento, toman lugar diferentes transformaciones físicas y químicas, tales como: pérdida de agua desde el alimento, migraciones de componentes solubles, migraciones del aceite al alimento, deformaciones mecánicas, reacciones químicas (reacción de Maillard), degradación de proteína, y finalmente, transformaciones fisicoquímicas (gelatinización, retrogradación, o transición vítrea de carbohidratos), (Saguy \& Pinthus, 1995; Vitrac et al., 2000; Yamsaengsung \& Moreira, 2002; Farinu \& Baik, 2007).

Uno de los principales objetivos del freído por inmersión es remover el agua de los alimentos. Por esta razón, predecir la pérdida de humedad es importante, no sólo para desarrollar un modelo para diseñar procesos de freído por inmersión sino también para controlarlo. Otro aspecto importante a considerar es la absorción de aceite, el cual está relacionado con la pérdida de agua (Saguy \& Pinthus, 1995).

Los modelos matemáticos son usados para el diseño y optimización de los procesos industriales. Para el caso del freído, muchos modelos con diferentes grados de complejidad han sido propuestos para describir el mecanismo de pérdida de humedad. Se han reportado métodos que asumen que el proceso puede ser descrito por la teoría de transferencia de masa, siendo controlada por los mecanismos de difusión interna. Farkas et al. (1995) han considerado dos regiones en el producto, la corteza y el corazón, descubriendo que los cambios en la velocidad de secado son debidos a los cambios en la velocidad de formación de la costra o corteza. Otros autores asumen que el efecto de la difusión del agua puede ser ignorado en procesos a altas temperaturas tales como el freído y que la transferencia de calor domina el proceso. Entre estos modelos, los difusivos son los que más fácilmente se formulan y se obtienen resultados muy acertados. La difusividad efectiva que aparece en estos modelos incluye el efecto de las hipótesis conocidas y los fenómenos desconocidos que no son considerados (Moyano \& Berna, 2002; Math et al., 2004; Tungsangprateep \& Jindal, 2004; Budzaki \& Seruga, 2005; Pedreschi et al., 2005; Torezan et al., 2007; Grenier et al., 2007).

Erdoğdu (2005) y Yildiz et al. (2007) plantearon una ecuación de difusión que describe la concentración como una función del tiempo y la posición para una placa infinita, por otro lado, el modelo empírico frecuentemente usado para describir la absorción de aceite durante el freído es el modelo propuesto por Moyano \& Pedreschi (2006), estos modelos son muy utilizados en la actualidad para describir el proceso de freído.

El objetivo de esta investigación fue evaluar los cambios humedad y absorción de aceite ocurridos en el freído por inmersión de rodajas de yuca (Manihot esculenta Crantz) variedad ICAcosteña sometidas a diferentes pre-tratamientos para la determinación de los parámetros cinéticos de los modelos matemáticos Moyano \& Pedreschi (2006) para absorción de aceite y la ley de Fick de la difusión para la humedad.

\section{Metodología}

\subsection{Materiales}

La yuca (variedad ICA costeña adquirida de un banco de germoplasma de CORPOICA, con $45 \%$ de sólidos secos aproximadamente) y aceite de palma (Caribe ${ }^{\circledR}$, Sincelejo, Colombia, producto de FAMAR) fueron las materias primas. El tubérculo se lavó y peló manualmente, posteriormente fue cortado en discos o rodajas de $3.5 \pm 0.03 \mathrm{~cm}$ de diámetro y $1.308 \pm 0.05 \mathrm{~mm}$ de espesor usando un cortador cilíndrico marca Hobart ${ }^{\circledR}$ modelo 
FP100 (Empresa Hallde, Suecia).

\subsection{Pretratamientos}

Los siguientes pretratamientos fueron usados: i) escaldado en agua caliente a $80{ }^{\circ} \mathrm{C}$ por $4 \mathrm{~min}$ y ii) osmodeshidratación en solución salina $3 \% \mathrm{p} / \mathrm{p}$ a $30{ }^{\circ} \mathrm{C}$ por $4 \mathrm{~h}$ y relación muestra/ solución de 1:20. Adicionalmente, se realizó el experimento en muestras control (sin escaldar ni osmodeshidratar).

\subsection{Condiciones de freído}

Las rodajas fueron procesadas en freído por inmersión en 2 litros de aceite en una freidora eléctrica (DUPREÉ $®$, USA) a temperaturas de $140^{\circ} \mathrm{C}, 160^{\circ} \mathrm{C}$ y $180^{\circ} \mathrm{C}$ y tres repeticiones. La relación peso de yuca/ $\mathrm{L}$ de aceite fue 0.005 . Las rodajas fueron freídas a diferentes intervalos de tiempo hasta un contenido final de humedad aproximado de $1 \%$ (en base seca).

\subsection{Análisis de humedad y aceite}

El contenido de humedad promedio de las rodajas de yuca, expresado como $\mathrm{g}$ de agua $\mathrm{g}^{-1}$ sólidos secos, se realizó por secado en estufa convectiva a $103{ }^{\circ} \mathrm{C}$ hasta peso constante (Método 984.25; AOAC, 2002). El contenido de aceite promedio de las rodajas de yuca, expresado como g aceite $\mathrm{g}^{-1}$ sólidos secos, se realizó por el método de extracción Soxhlet (AOAC, 1984). La precisión de la medida fue de $0.0003 \mathrm{~g}$ y un nivel de confianza de 0.95 .

\subsection{Diseño experimental}

El experimento fue conducido bajo un diseño en bloque completamente al azar con arreglo factorial $3^{2}$ (factores: temperatura y pre-tratamientos) con tres repeticiones. El nivel de significancia utilizado fue del $5 \%$.

\subsection{Análisis estadístico de datos}

A los errores experimentales se evaluaron los supuestos de aleatoriedad, linealidad, normalidad y homogeneidad de varianzas. Comprobados los supuestos, a las variables de respuesta se realizaron
Análisis de varianzas, test de diferencias de medias (Tukey) y ajuste de regresiones no lineales (método de Marquardt) con significancia del 5\%.

Para la obtención de los parámetros cinéticos se realizaron los siguientes procedimientos:

\section{Cinética de pérdida de humedad:}

La ley de Fick para placas infinitas truncada en el primer término (para $\mathrm{Fo}>0.2$ ) e integrada con respecto a todo su volumen, cuya resolución fue propuesta por Erdoğdu (2005) y Yildiz et al. (2007) y se describe a continuación, fue utilizada para estimar la difusividad efectiva de transferencia de agua y el coeficiente convectivo de transferencia:

$$
\frac{\bar{M}(t)-M_{\infty}}{M_{i}-M_{\infty}}=\frac{2 \operatorname{sen}^{2} \mu_{1}}{\mu_{1}\left[\mu_{1}+\operatorname{sen} \mu_{1} \cos \mu_{1}\right]} e^{-\mu_{1}^{2} \frac{D_{e f f} t t}{L^{2}}}(1)
$$

Donde: $t$ es el tiempo de freído, $D_{\text {eff }}$ es la difusividad efectiva de la humedad, L es la mitad del espesor de la placa, $\mu_{1}$ es la primera raíz de la solución, $\bar{M}(t)$ es la concentración media de agua (g agua/g de sólidos) en el sólido, $M_{i}$ es la concentración de agua ( $g$ agua/g de sólidos) inicial en el sólido y $M_{\infty}$ es la concentración de agua ( $\mathrm{g}$ agua/g de sólidos) en el equilibrio que para el freído se puede estimar de valor cero (0).

Experimentalmente se tienen datos de $\bar{M}(t)$ y $t$, por tanto se determina la $D_{\text {eff }} \mathrm{y}$ de forma adicional $\mu_{1}$ ajustando los datos experimentales a la Ec. (1) por regresión no lineal. A partir de la definición del número de Biot de transferencia de masa $\left(B i_{m}\right)$ es obtenido el coeficiente efectivo de transferencia de masa $\left(k_{c}\right)$ :

$$
B i_{m}=\mu_{1} \tan \mu_{1}=\frac{k_{c} L}{D_{e f f}}
$$

La relación para la variación de la difusividad efectiva con la temperatura de freído $T$ fue descrita usando una relación tipo Arrhenius, y la relación del coeficiente convectivo de transferencia de masa con la temperatura de freído $T$ fue descrita por la 
ecuación lineal.

Cinética de absorción de aceite:

Para describir la absorción de aceite durante el freído se utilizó el modelo propuesto por Moyano \& Pedreschi (2006):

$$
O=\frac{O_{e q} K t}{1+K t}
$$

Donde $O$ es el contenido de aceite en el tiempo t (aceite libre, en base seca), $O_{e q}$ es el contenido de aceite en el equilibrio (o contenido máximo) (aceite libre, en base seca) al tiempo $t=\infty, K$ representa la velocidad específica de absorción de aceite. En el modelo, al $t=0$, el contenido de aceite es nulo, y para tiempos largos, el contenido de aceite alcanza el valor del equilibrio.

La relación para la variación del contenido de aceite en el equilibrio, $O_{e q}$, con la temperatura de freído $T$ se puede describir usando una relación tipo Arrhenius para obtener la energía de activación (Bravo et al., 2009).

\section{Resultados y Discusión}

\subsection{Cinética de transferencia de masa-pérdida de humedad}

La Figura 1 muestra la evolución del contenido de humedad (como humedad adimensional M/M0, donde $\mathrm{M}$ es el contenido de humedad promedio en base seca al tiempo t y M0 es el contenido de humedad promedio en base seca inicial) de las rodajas de yuca durante el proceso freído por inmersión a $140^{\circ} \mathrm{C}, 160^{\circ} \mathrm{C}$ y $180^{\circ} \mathrm{C}$ de la muestra control (comportamiento semejante se presentan con las muestras con tratamiento de escaldado y con tratamiento de osmodeshidratación). En esta figura, los puntos representan los valores experimentales y la línea continua son los valores calculados con el modelo de difusión. Las curvas muestran las formas típicas de las curvas de secado y son similares a las reportadas por los procesos de freído por inmersión de otros productos (Pedreschi et al., 2005; Vitrac et al., 2002; Sahin et al., 1999; Krokida et al., 2001b). Además, se observa que la velocidad de deshidratación depende de la temperatura, es decir, cuando la temperatura incrementa en un tiempo dado, el contenido de humedad residual en el producto disminuye. Adicionalmente, la velocidad de pérdida de agua incrementa cuando la temperatura del aceite aumenta, esto es una indicación de la ausencia de la influencia de la formación de la costra o corteza (Budzaki \& Seruga, 2005).

En la tabla 1, se muestran los valores de la difusividad efectiva en función de la temperatura, $\mathrm{y}$ el coeficiente de determinación $\left(\mathrm{R}^{2}\right)$. Puede ser observado que los valores del coeficiente de

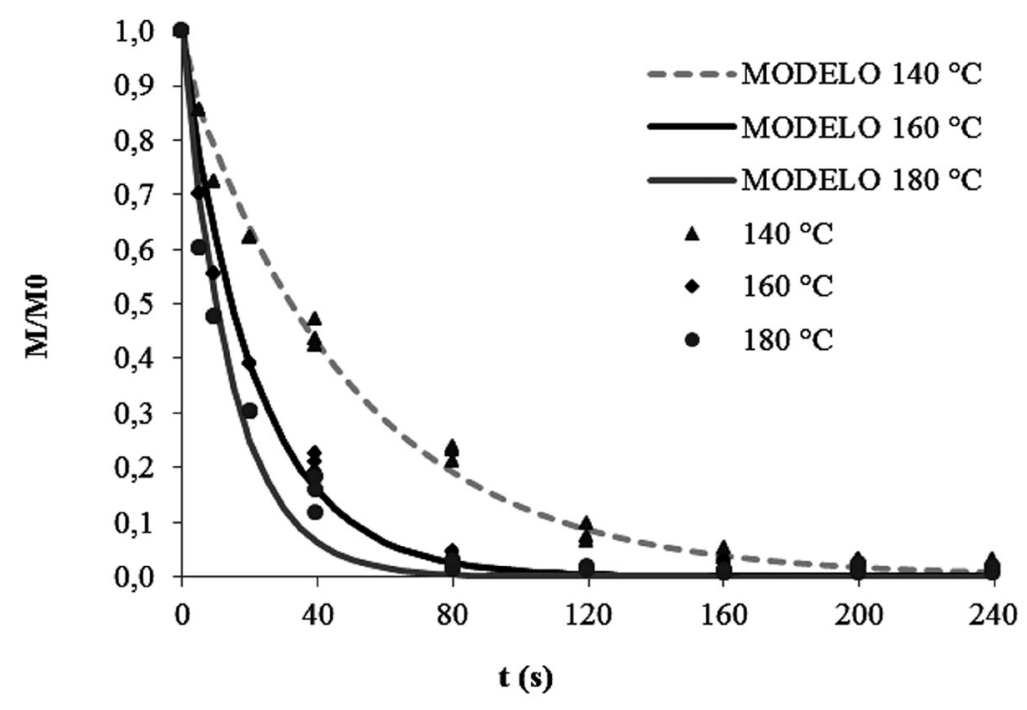

Figura 1. Curvas de humedad adimensional versus tiempo para muestra control. 
Tabla 1. Coeficiente de difusividad efectiva y coeficiente de transferencia de masa para el modelo de Fick, Energía de activación del modelo de Arrhenius para la $D_{\text {eff }}$

\begin{tabular}{|c|c|c|c|c|c|c|}
\hline TRATAMIENTO & $T\left({ }^{\circ} \mathrm{C}\right)$ & $D_{e f f}\left(10^{-9} \mathrm{~m}^{2} / \mathrm{s}\right)$ & $K_{c}\left(10^{-5} \mathrm{~m} / \mathrm{s}\right)$ & $R^{2}$ & $E a(k J / m o l)$ & $R^{2}$ \\
\hline \multirow{3}{*}{ CONTROL } & 140 & $10.44 \pm 0.22$ & $4.41 \pm 0.31$ & 0.994 & \multirow{3}{*}{$37.84 \pm 0.898$} & \multirow{3}{*}{0.999} \\
\hline & 160 & $17.02 \pm 0.27$ & $5.26 \pm 0.21$ & 0.994 & & \\
\hline & 180 & $27.62 \pm 0.15$ & $6.03 \pm 0.09$ & 0.997 & & \\
\hline \multirow{3}{*}{ ESCALDADO } & 140 & $20.60 \pm 0.15$ & $1.47 \pm 0.02$ & 0.998 & \multirow{3}{*}{$41.80 \pm 1.399$} & \multirow{3}{*}{0.969} \\
\hline & 160 & $42.49 \pm 0.16$ & $2.10 \pm 0.11$ & 0.997 & & \\
\hline & 180 & $60.00 \pm 0.03$ & $2.23 \pm 0.04$ & 0.999 & & \\
\hline \multirow{3}{*}{ OSMODESHIDRATACIÓN } & 140 & $34.63 \pm 0.12$ & $4.86 \pm 0.11$ & 0.995 & \multirow{3}{*}{$56.95 \pm 1.896$} & \multirow{3}{*}{0.960} \\
\hline & 160 & $96.21 \pm 0.09$ & $5.84 \pm 0.06$ & 0.980 & & \\
\hline & 180 & $148.00 \pm 0.08$ & $7.37 \pm 0.13$ & 0.973 & & \\
\hline
\end{tabular}

determinación son mayores que 0.97 , por tanto, se puede afirmar que los valores experimentales y los valores calculados están muy bien correlacionados y que el modelo de difusión de Fick describe satisfactoriamente la transferencia de agua en el proceso de freído. Los valores de difusividad efectiva hallados en este trabajo son más altos que los reportados en la literatura, sin embargo, están en el orden de magnitud para los alimentos deshidratados $10^{-9}$ a $10^{-11}$ (Bravo et al., 2009; Saravacos \& Maroulis, 2001). Algunos valores de la difusividad efectiva para otros materiales deshidratados por freído por inmersión han sido reportados en la literatura, por ejemplo, para papas a la francesa freídas a $160^{\circ} \mathrm{C}, 170^{\circ} \mathrm{C}$ y $180{ }^{\circ} \mathrm{C}$ los valores obtenidos fueron $4.14,5,24$ y $6.7 \times 10^{-9} \mathrm{~m}^{2} / \mathrm{s}$, respectivamente (Moyano \& Berna, 2002). Pedreschi et al. (2005) reportaron que la $D_{\text {eff }}$ del agua durante el freído convencional de chips de papas pretratadas está entre $13 \times 10^{-9} \mathrm{y}$ $9.8 \times 10^{-9} \mathrm{~m}^{2} / \mathrm{s}$, siendo estos valores menores que los del freído de yuca obtenidos en este trabajo dado a composición y a la microestructura de la yuca (Saravacos \& Maroulis, 2001).

Los pretratamientos afectaron significativamente la $\mathrm{D}_{\text {eff }}(\mathrm{p}<0.05)$, siendo mayores los valores en las muestra $\mathrm{s}$ osmodeshidratadas que en las muestras escaldadas y de control a una misma temperatura de freído, esto es explicado por Saravacos \& Maroulis (2001), ya que, para alimentos con contenido alto de humedad, la difusión de líquidos y el flujo capilar pueden ser los principales mecanismos de transportes (relativa baja porosidad y baja difusividad) como es el caso de muestras control y escaldadas; en contenidos de humedad intermedia, el agua puede ser transportada por flujo capilar y difusión del vapor lo cual incrementa los valores de la difusividad efectiva, como es el caso de muestras osmodeshidratadas. En esta situación, los poros y canales permiten el transporte más fácil del agua.

Los valores de $D_{\text {eff }}$ mostrados en la tabla 1 fueron ajustados al modelo de Arrhenius. El logaritmo natural de $D_{\text {eff }}$ versus la inversa de la temperatura absoluta mostraron una tendencia lineal, con un coeficiente de determinación entre $0.9596 \mathrm{y}$ 0.9994. La energía de activación para procesos de difusión es un buen indicador del mecanismo predominante; energías de activación altas son halladas comúnmente en materiales con contenido de humedad baja debido a una fuerte interacción de agua-sustrato como es el caso de las muestras osmodeshidratadas (Saravacos \& Maroulis, 2001; Troncoso \& Pedreschi, 2009).

La tabla 2 muestra los datos del coeficiente convectivo de transferencia de masa en función a la temperatura, el cual tiene un comportamiento lineal en los tres tratamientos con un buen ajuste estadístico de los datos. De forma adicional se observa que el coeficiente convectivo másico aumenta al aumentar la temperatura del aceite de freído y que los valores de $K_{c}$ son mayores en las muestras con pre-tratamiento de osmodeshidratación seguidas por las muestras 
Tabla 2. Modelo lineal del Coeficiente convectivo de transferencia de masa

\begin{tabular}{ccc}
\hline TRATAMIENTO & Modelo lineal & $\boldsymbol{R}^{2}$ \\
\hline CONTROL & $K_{c}=4.050 \times 10^{-7 *} T\left({ }^{\circ} \mathrm{C}\right)-1.250 \times 10^{-5}$ & 0.9996 \\
ESCALDADO & $K_{c}=1.900 \times 10^{-7 * T}\left({ }^{\circ} \mathrm{C}\right)-1.107 \times 10^{-5}$ & 0.8739 \\
OSMODESHIDRATACIÓN & $K_{c}=6.275 \times 10^{-7 *} T\left({ }^{\circ} \mathrm{C}\right)-4.017 \times 10^{-5}$ & 0.9843 \\
\hline
\end{tabular}

control y las muestras escaldadas. Este comportamiento de las muestras escaldadas $\left(K_{c}\right.$ más bajos) se debe a que en la superficie de la rodaja se encuentra almidón gelificado que fácilmente se desprende ante la transferencia de agua al medio aportando una resistencia adicional al paso de agua del sólido al aceite (Saravacos \& Maroulis, 2001; Troncoso \& Pedreschi, 2009).

\subsection{Cinética de transferencia de masa- absorción de aceite}

La Figura 2 muestra el contenido de aceite durante el tiempo de freído para las muestras de yuca sin pretratamiento (control), a las temperaturas de $140{ }^{\circ} \mathrm{C}, 160^{\circ} \mathrm{C}$ y $180^{\circ} \mathrm{C}$. Se observa que para un mismo tiempo de proceso a medida que disminuye la temperatura del aceite el contenido de aceite final en la rodaja de yuca se incrementa, este comportamiento es semejante con las muestras con tratamiento de escaldado y con tratamiento de osmodeshidratación y está de acuerdo con lo reportado en otras investigaciones (Moyano \& Pedreschi, 2006; Kita \& Lisińska, 2005; Kita et al., 2007).

La tabla 3 muestra los parámetros del modelo cinético de Moyano y Pedreschi calculado para las diferentes condiciones experimentales, los datos experimentales del aceite absorbido durante el freído ajustaron adecuadamente el modelo cinético empírico estudiado con valores altos del coeficiente de determinación. Además, se observa que los valores de la velocidad específica $(\mathrm{K})$ incrementa con el aumento de la temperatura de freído. Por otro lado, en todos

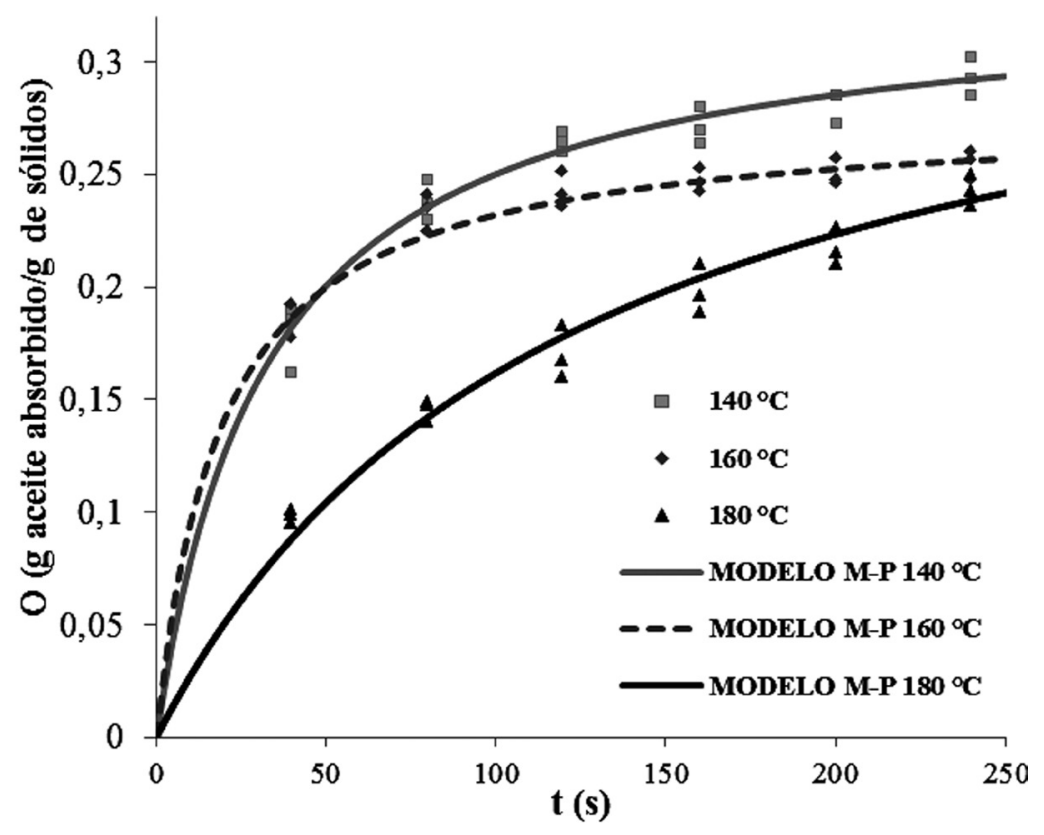

Figura 2. Absorción de aceite durante el freido para rodajas de yuca muestra control. 
Tabla 3. Parámetros del modelo Moyano y Pedreschi y Energía de activación del modelo de Arrhenius del contenido de aceite en el equilibrio $\left(O_{e q}\right)$.

\begin{tabular}{ccccccc}
\hline TRATAMIENTO & $\boldsymbol{T}\left({ }^{\circ} \boldsymbol{C}\right)$ & $\boldsymbol{O}_{e q}$ & $\boldsymbol{K}\left(10^{-3} \boldsymbol{s}^{-1}\right)$ & $\boldsymbol{R}^{2}$ & $\boldsymbol{E a}(\boldsymbol{k J} / \mathbf{m o l})$ & $\boldsymbol{R}^{2}$ \\
\hline \multirow{2}{*}{ CONTROL } & 140 & $0.3607 \pm 0.012$ & $8.14 \pm 0.0007$ & 0.989 & & \\
& 160 & $0.3321 \pm 0.005$ & $30.52 \pm 0.0021$ & 0.995 & $-10.25 \pm 2.52$ & 0.947 \\
& 180 & $0.2767 \pm 0.003$ & $51.92 \pm 0.0028$ & 0.992 & & \\
ESCALDADO & 140 & $0.3003 \pm 0.005$ & $28.5 \pm 0.0018$ & 0.990 & & \\
& 160 & $0.2814 \pm 0.004$ & $50.79 \pm 0.0034$ & 0.991 & $-2.77 \pm 0.66$ & 0.840 \\
& 180 & $0.2799 \pm 0.004$ & $106.23 \pm 0.0114$ & 0.986 & & \\
OSMODESHIDRATACIÓN & 140 & $0.2444 \pm 0.002$ & $50.29 \pm 0.0026$ & 0.994 & & \\
& 160 & $0.2189 \pm 0.006$ & $54.96 \pm 0.0081$ & 0.960 & $-17.22 \pm 3.75$ & 0.906 \\
& 180 & $0.1564 \pm 0.006$ & $77.08 \pm 0.0080$ & 0.950 & & \\
\hline
\end{tabular}

los casos, el contenido de aceite en el equilibrio $\left(O_{e q}\right)$ disminuye cuando la temperatura del aceite aumenta y su efecto sobre el contenido de aceite en el equilibrio fue significativo $(\mathrm{p}<0.05)$, lo cual está de acuerdo con lo reportado por Moyano \& Pedreschi (2006).

Adicionalmente, la tabla 3 muestra los valores de la energía de activación negativa del contenido de aceite en el equilibrio para el modelo cinético de absorción de aceite utilizado en este estudio, indicando que el efecto de la temperatura sobre el contenido de aceite en el equilibrio es mayor en las muestras osmodeshidratadas que en las muestras escaldadas concordando con lo reportado por Moyano \& Pedreschi (2006) en freído de rodajas de papas sometidas a pretratamientos de escaldado y osmodeshidratación.

El análisis de varianza del aceite absorbido y el test de comparación de medias (Tukey) mostraron que el escaldado y la osmodeshidratación tuvieron un efecto significativo $(\mathrm{p}<0.05)$ sobre el contenido de aceite en el equilibrio de las rodajas de yuca para una misma temperatura, siendo el contenido menor en las muestras osmodeshidratadas seguido por las muestras escaldadas, comparadas con la muestra control. Es decir, que para una misma temperatura del aceite de freído, el contenido de aceite final en la rodaja de yuca es mayor en la muestra control, es posible que esto se deba a que en las muestras escaldada y osmodeshidratada se logra un mejor desarrollo de la costra o corteza, la cual actuaría como una barrera para la absorción de aceite de acuerdo con lo reportado en otras investigaciones de freído de papas a la francesa y rodajas de papas (Moyano \& Pedreschi, 2006; Kita \& Lisińska, 2005; Kita et al., 2007). El escaldado ha sido reportado como un pretratamiento que puede reducir la absorción de aceite debido a la gelatinización del almidón de la superficie (Rimac-Brncic et al., 2004). Resultados similares han sido reportados en el escaldado y freído de muestras de papas (Troncoso \& Pedreschi, 2009). Por otro lado, el uso de la deshidratación osmótica como pre-tratamiento afecta el contenido final de aceite absorbido en el producto como resultado del contenido de humedad inicial del producto y la gelatinización parcial de las moléculas de almidón en la muestra después del freído (Ikoko \& Kuri, 2007). Otro factor que puede ser responsable de la reducción del contenido de aceite en las muestras tratadas por deshidratación osmótica incluye la infusión de material sólido dentro de la matriz del alimento cuando el contenido de humedad es reducido (Krokida et al., 2001a; Krokida et al., 2001b). Algunos autores han reportado que la deshidratación osmótica ha sido un pretratamiento útil para reducir la absorción de aceite en las papas fritas (Krokida et al., 2001a; Krokida et al., 2001b), concordando con los resultados obtenidos en este trabajo.

\section{Conclusiones}

El escaldado y la osmodeshidratación afectaron 
significativamente a la Difusividad efectiva de transferencia de masa $(\mathrm{p}<0.05)$. A una misma temperatura de aceite de freído los valores mayores estuvieron en las muestras osmodeshidratadas.

La difusividad efectiva incrementó con la temperatura del aceite de forma exponencial y fue modelada con la ecuación de Arrhenius, mientras que el coeficiente convectivo de transferencia de masa aumentó linealmente con la temperatura del aceite y además los valores fueron mayores en las muestras con osmodeshidratación, seguido de las muestras control y escaldadas.

Para todos los tratamientos, el aceite absorbido incrementó con el tiempo de freído y con la disminución de la temperatura del aceite de freído siendo mayor para las rodajas muestra control.

Los valores de la velocidad específica de absorción de aceite $(K)$ incrementaron con el aumento de la temperatura de aceite de freído mientras que el contenido de aceite en el equilibrio $\left(O_{e q}\right)$ disminuyó siendo menor en las muestras osmodeshidratadas.

\section{Referencias bibliográficas}

AOAC. ( 1984). AOAC official method 7.062. Official methods of analysis of AOAC international. The Association of Official Analytical Chemists International, Gaithersburg, MD.

AOAC. (2002). AOAC official method 984.25, 17th ed. Official methods of analysis of AOAC international, vol. 2. The Association of Official Analytical Chemists International, Gaithersburg, MD.

Bassama, J.; Brat, P.; Boulanger, R.; Günata, Z. \& Bohuon, P. (2012). Modeling deep-fat frying for control of acrylamide reaction in plantain. Journal of Food Engineering, 113, 156-166.

Bravo, J., Sanjuán, N., Ruales, J. \& Mulet, A. (2009). Modeling the dehydration of apple slices by deep fat frying. Drying Technology: An International Journal, 27 (6), 782-786.
Budzaki, S. \& Seruga, B. (2005). Moisture loss and oil uptake during deep fat frying of "krostula" dough. European Food Research and Technology, 220, 90-95.

Erdoğdu, F. (2005).Mathematical approaches for use of analytical solutions in experimental determination of heat and mass transfer parameters. Journal of Food Engineering, 68, 233-238.

Farinu, A. \& Baik, O.-D. (2007). Heat transfer coefficients during deep fat frying of sweet potato: Effects of product size and oil temperature. Food Research International, 40, 989-994.

Farkas, B.E., Singh, R.P. \& Rumsey, T.R. (1995). Modeling heat and mass transfer in immersion frying.II, Model solution and verification.Journal of Food Engineering, 29, 227-248.

Grenier, D., Bohuon, P., Me'ot, J.-M., Lecomte, D. \& Baille'res, H. (2007). Heat and mass transfer in fry drying of wood. Drying Technology, 25 (3), 511-518.

Ikoko, J. \& Kuri, V. (2007). Osmotic pretreatment effect on fat intake reduction and eating quality of deep-fried plantain. Food Chemistry, 102, 523-531.

Kita, A., Lisińska, G. \& Golubowska, G. (2007). The effects of oils and frying temperature on the texture and fat content of potato crisps. Food Chemistry, 102, 1-5.

Kita, A. \& Lisińska, G. (2005). The influence of oil type and frying temperatures on the texture and oil content of French fries.Journal of the science of Food and Agriculture, 85, 2600-2604.

Krokida, M., Oreopoulou, V., Maroulis, Z. \& Marinos-Kouris, D. (2001a). Deep fat frying of potato strips quality issues. Drying technology, 19, 879-935.

Krokida, M., Oreopoulou, V., Maroulis, Z. \& Marinos-Kouris, D. (2001b). Effect of osmotic dehydration pretreatment on quality of French fries.Journal of Food Engineering, 49,339-345. 
Math, R.G., Velu, V., Nagender, A. \& Rao, D.G. (2004). Effect of frying conditions on moisture, fat and density of papad. Journal of Food Engineering, 64, 429-434.

Moyano, P.C. \& Berna, A.Z. (2002). Modeling water loss during frying of potato strips: Effect of solute impregnation. Drying Technology, 20 (7), 1303-1318.

Moyano, P. \& Pedreschi, F. (2006). Kinetics of oil uptake during frying of potato slices: Effect of pre-treatments. Lebensmittel-Wissenschaft undTechnologie, 39, 285-291.

Pedreschi, F., Hernández, P., Figueroa, C. \& Moyano, P. C. (2005). Modeling water loss during frying of potato slices. International Journal of Food Properties, 8, 289-299.

Rimac-Brncic, S.; Lelas, V.; Rade, V. \& Simundic, B. (2004). Decreasing of oil absorption in potato strips during deep fat frying. Journal of Food Engineering,64, 237-241.

Saguy, I.S. \& Pinthus, E.J. (1995). Oil uptake during deep-fat frying: Factors and mechanism. Food Technology,49, 142-145.

Sahin, S., Sastry, S. \& Bayindirli, L. (1999). The determination of convective heat transfer coefficient during frying. Journal of Food Engineering,39, 307-311.

Saravacos, G. D. \& Maroulis, Z. B. (2001). Transport properties of foods. New York: Marcel Dekker.
Torezan, G.A.P., Menezes, H.C., Katekawa, M.E. \& Silva, M.A. (2007). Microstructure and adsorption characteristics of mango chips obtained by osmotic dehydration and deep fat frying. Drying Technology, 25 (1), 153-159.

Troncoso, E. \& Pedreschi, F. (2009). Modeling water loss and oil uptake during vacuum frying of pretreated potato slices. LWT-Food Science and technology, 42, 1164-1173.

Tungsangprateep, S. \& Jindal, V.K. (2004). Sorption isotherms and moisture diffusivity in fried cassava-shrimp chips. International Journal of Food Properties, 7 (2), 215-227.

Vitrac, O., Trystram, G. \& Raoult-Wack, A-L. (2000). Deep-fat frying of food: Heat and mass transfer, transformations and reactions inside the frying material. European Journal of Lipid Science and Technology, 102, 529-538.

Vitrac, O., Dufour, D., Trystram, G. \& RaoultWack, A-L. (2002). Characterization of heat and mass transfer during deep-fat frying and its effect on cassava chip quality. Journal of Food Engineering, 53, 161-176.

Yamsaengsung, R. \& Moreira, R.G. (2002). Modelling the transport phenomena and structural changes during deep fat frying.Part I. model development. Journal of Food Engineering, 53, 1-10.

Yildiz, A., Palazoğlu, T. \& Erdoğdu, F. (2007). Determination of heat and mass transfer parameters during of potato slices. Journal of Food Engineering,79, 11-17. 